\title{
Analytical results for rotating and linear magnetic brakes
}

\author{
José Arnaldo Redinz \\ Departamento de Física, Universidade Federal de Viçosa 36570-000, Viçosa, MG - Brazil \\ E-mail: redinz@ufv.br
}

\begin{abstract}
A theory of magnetic damper (eddy-current brake) which can be applied to axial-flux rotating and linear systems moving under the influence of an arbitrary arrangement of magnetic poles is derived from basic electromagnetic principles. Analytical expressions for the braking forces and torques are obtained in the low-velocity limit of the moving nonmagnetic conductor.
\end{abstract}

\section{Introduction}

When a nonmagnetic conductor moves exposed to a constant external magnetic field, eddy currents may be induced in the material. The interaction between the induced eddy currents and the external magnetic field leads to a force on the conductor that opposes its motion. This drag force occurs in many practical devices such as motors, magnetic bearings, magnetically levitated vehicles and brakes.The magnetic brake is widely used in practice in measuring instruments, electrical machines, cars, trains, roller coasters, exercise bicycles, vibration dampers in cantilever beams, satellites and spacecrafts and in other devices. Some advantages of the magnetic brakes over other brake systems are: i) they have lower sensitivity to environmental parameters, such as temperature and aging; ii) they are free from viscous fluids, seals and periodic maintenance; iii) they can be considered a green - environmental friendly - technology because they are also free from wear debris. These advantages make the magnetic brakes well suited and promising for use in space technology.

Popular physics lecture demonstrations related to the magnetic brake are that of a magnet falling inside a cylindrical conductor, e.g., a copper pipe $[1,2]$, and the one of a magnet fixed to a glider sliding on an air track $[3,4]$. To understand magnetic braking we can start with a simple model made up of an infinite thin metal strip moving under the field of a rectangular magnetic pole [5]. The braking force is obtained from the Lorentz force on the eddy currents, assuming that the current density is uniform inside the region of the magnet 'footprint'. Later improved theories take into account the induced electric fields and space charges which arise when conductors move in a magnetic field. These space charges and the fields associated with them have been studied in a number of conducting systems rotating in static and uniform magnetic fields (see, for example, $[6,7,8]$ ). Depending on the geometries of the conductor and the magnetic field, these space charges may be accompanied by an electric current density $\vec{J}$, as in the case of the Faraday's first dynamo [9, 10] and the induction motor [11]. References to these space charges on a magnetic brake made up of a disk rotating under the magnetic field of a single pole can be found in literature, $[12,13,14,15,16,17]$ in the context of analytical calculations of forces and torques.

The purpose of this paper is to describe an analytical approach which is sufficiently general to compute the braking forces and torques on magnetic brakes made up of a nonmagnetic conductor which can be a rotating disk, a rotating ring or a linearly moving strip, under the influence of an arbitrary arrangement of magnetic poles. In contrast to previous works, our approach starts from the analysis of the space charges induced on a conductor moving in a magnetic field. These space charges induced inside $\left(\rho_{F}\right)$ and at the borders $\left(\sigma_{F}\right)$ of the pole projection area and at the conductor external surface $\left(\sigma_{A}\right)$ are the sources of the electric field $\vec{E}$ inside the conductor which, together with the Lorentz force $\vec{v} \times \vec{B}$, drives the eddy currents responsible for the braking forces. We disregard the magnetic field due to the eddy currents. The present analysis is therefore valid for the case of low relative velocities between the magnets and the moving conductor, for which the brake force is proportional to the speed and the magnetic brake acts as a linear damper.

Previous analytical works on this subject either completely ignore the space charges induced on the disk, due to the technique used in the solution $[14,18,19,20]$ or due to the simplicity of the model [5], or calculate these space charges only partially. According to the convenience, only $\rho_{F}$ is calculated, ignoring the surface charge density $\sigma_{F}$ [17] or, conversely, only $\sigma_{F}$ is calculated ignoring the charge density $\rho_{F}[12,13,15,16]$. The charge density at the external surface of the moving conductor $\left(\sigma_{A}\right)$ is never mentioned in these works. The calculation of these space charges is essential for the correct description and understanding of the current drifting in terms of the Lorentz force and the relation between the magnetic brake operation and electrostatics.

We start calculating the induced charge densities on a rotating thin disk due to the motional electromotive force caused by the magnetic field of one circular magnetic pole. From the charge densities we obtain solutions of the Laplace equation and use the method of images to compute the electric potential inside the disk which satisfies the boundary conditions. The presence of a second independent magnetic pole is considered and the braking torques due to one pole and due to the interaction between the poles 
are obtained from the current density. In a second step we consider a rotating thin metal ring, satisfying the new boundary conditions by applying the method of images for an infinite set of image charges. This geometry was not considered in previous works on the subject, but it is an useful geometry for dampers because of its easily adjustable moment of inertia [21]. Finally, in the third step we consider a linearly moving thin metal sheet as a limit geometry of the ring with infinite radius. The braking forces on the sheet due to two magnetic poles are obtained from the expressions of the torques on the ring.

\section{Results}

\subsection{A rotating conducting disk}

\subsubsection{The current density $\vec{J}$}

We consider a rotating nonmagnetic conducting disk which rotates with angular speed along the $z$-axis: $\vec{\omega}=\omega \hat{z}$. The disk has radius $A$, thickness $h$, electrical conductivity $\sigma$, permeability $\mu_{0}$ and permitivity $\epsilon$. We assume a thin disk and a small velocity of rotation, such that skin effects and the magnetic field due to the conduction and convection currents on the disk can be neglected. In the axis rest frame the electric field is $\vec{E}(\vec{r})$, the magnetic field is the applied field $\vec{B}(\vec{r})$, and the conduction current density is $\vec{J}(\vec{r})$. A point inside the disk moves at a velocity $\vec{v}(\vec{r})=\omega \rho \hat{\phi}$ in relation to the axis. All the quantities we calculate are assumed to be stationary, which implies that $\vec{\nabla} \cdot \vec{J}=0$, and uniform throughout the disk thickness (coordinate $z$ ) with null components along the $z$-axis.

We consider a break with two magnetic poles. The applied magnetic field of any pole, $\vec{B}=B \hat{z}$, is uniform inside a circular tube of radius $a$ with center located at a distance $c$ from the disk axis. For pole 1 , with center at $(x=c, y=0)$ (which is shown as a solid circle in Fig. 1) we define the two regions: Region I is the disk material where the applied field $B=0$, it is given by $\rho<A$ with exception of region II. Region II is the disk material where the applied field $B \neq 0$, it is given by $s<a$. Besides, Region III is the vacuum outside the cylinder, it is given by $\rho>A$. The boundary of a pole 2 which is displaced by $\alpha$ and has an applied magnetic field $\vec{B}_{2}$ is shown as a dotted circle in Fig. 1.

We will initially consider just one magnetic pole with field $\vec{B}_{1}=B_{1} \hat{z}$ in Region II.

We start by examining the dielectric response of the medium. The constitutive equation for the polarization $\vec{P}(\vec{r})$ inside the moving cylinder, in the first-order theory $\left((\omega A)^{2}<<c^{2}, c\right.$ is the speed of light), is (see, for example, Ref. [22]):

$$
\vec{P}(\vec{r})=\left(\epsilon-\epsilon_{0}\right)\left[\vec{E}(\vec{r})+\vec{v}(\vec{r}) \times \overrightarrow{B_{1}}(\vec{r})\right] .
$$

The constitutive equation for the conduction current density $J(\vec{r})$ (Ohm's law) has the form

$$
\vec{J}(\vec{r})=\sigma\left[\vec{E}(\vec{r})+\vec{v}(\vec{r}) \times \overrightarrow{B_{1}}(\vec{r})\right] .
$$

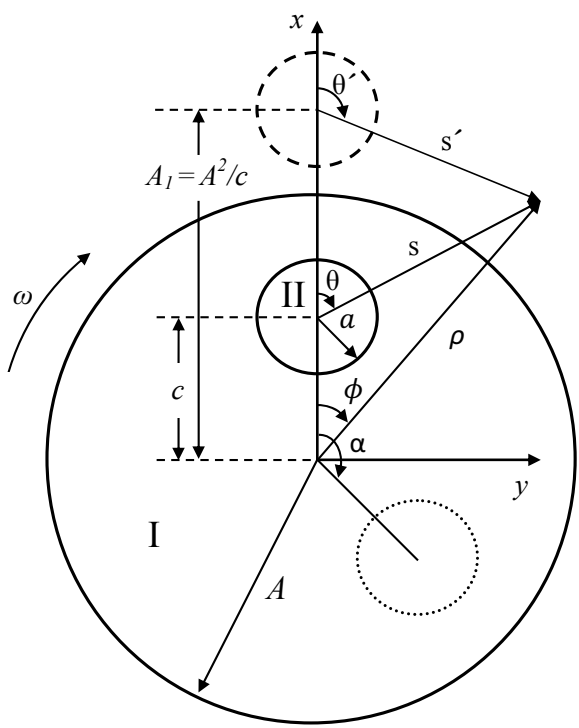

Figure 1: Plan view of the disk showing the axis, the polar coordinates we use in our calculations, the dimensions, and the Regions I and II for pole 1. The solid circle is the boundary of region II where the applied magnetic field of the first pole is uniform. The dashed circle refers to the boundary of the image cylinder defined in the text. The dotted circle is the boundary of a second magnetic pole displaced in space by an angle $\alpha$ from the first pole.

The reason for Eqs. 1 and 2 is that inside the moving material the force responsible for distorting the electronic orbitals of the atoms (polarization) and for the drift of the charge carriers (current) is $q\left(\vec{E}+\vec{v} \times \overrightarrow{B_{1}}\right)$ instead of $q \vec{E}$.

At steady state $\vec{\nabla} \cdot \vec{J}(\vec{r})=0$, and it follows that the space charge density of bound charges is zero, since $\vec{\nabla}$. $\vec{P}(\vec{r})=0$. From Gauss law we obtain the volume free charge density $\rho_{F}(\vec{r})$ given by:

$$
\rho_{F}(\vec{r})=\epsilon_{0} \vec{\nabla} \cdot \vec{E}(\vec{r})=-\epsilon_{0} \vec{\nabla} \cdot\left(\vec{v}(\vec{r}) \times \overrightarrow{B_{1}}(\vec{r})\right),
$$

which means that the $\vec{v}(\vec{r}) \times \overrightarrow{B_{1}}(\vec{r})$ field piles up the free charges at points in the conducting material where its divergence is non null. Thus, we have a uniform negative charge density in region II:

$$
\rho_{F}(\vec{r})=\left\{\begin{array}{l}
-2 \epsilon_{0} \omega B_{1} \text { in region II } \\
0 \text { in Region I . }
\end{array}\right.
$$

There is also a surface charge density $\sigma_{F}(\vec{r})$ at the boundary between regions I and II which can be obtained from the continuity condition for a field inside the material disk:

$$
\begin{aligned}
\sigma_{F}(\vec{r}) & =\epsilon_{0}\left[\left(-\vec{v}(\vec{r}) \times \vec{B}_{1}(\vec{r})\right)_{\mathrm{I}}-\right. \\
& \left.-\left(-\vec{v}(\vec{r}) \times \vec{B}_{1}(\vec{r})\right)_{\mathrm{II}}\right] \cdot \hat{n},
\end{aligned}
$$

where the unit normal vector $\hat{n}$ points from region II to region I. Thus, at the boundary $s=a$ between regions I and 
II we obtain a charge density:

$$
\begin{aligned}
\sigma_{F}(\vec{r}) & =\epsilon_{0}\left[0-\left(-\vec{v}(\vec{r}) \times \vec{B}_{1}(\vec{r})\right)_{\mathrm{II}}\right] \cdot \hat{s}= \\
& =\epsilon_{0} \omega B_{1}(a+c \cos \theta)=\sigma_{U}+\sigma_{N U}(\theta)
\end{aligned}
$$

where we defined $\sigma_{U}=\epsilon_{0} \omega B_{1} a$, a uniform charge density, and $\sigma_{N U}(\theta)=\epsilon_{0} \omega B_{1} c \cos \theta$, a non-uniform charge density at this boundary. Note that the bulk charge density $\rho_{F}$ and these surface charge densities make the disk electrically neutral.

There is also an initially unknown surface charge $\sigma_{A}(\phi)$ on the disk surface $\rho=A$ which cannot be obtained form a relation such as that in Eq. 5 because the field $\vec{v} \times \vec{B}_{1}$ is not known in vacuum.

The electric field $\vec{E}_{0}(\vec{r})$ due only to these known space charges satisfies $\vec{\nabla} \cdot \vec{E}_{0}=\rho_{F} / \epsilon_{0}$ in Region II and $\vec{\nabla} \times \vec{E}_{0}=$ 0 . Since this field is conservative, we can define an electric potential $V_{0}(\vec{r})$ (such that $\vec{E}_{0}=-\vec{\nabla} V_{0}$ ) which can be easily obtained:

$$
\begin{aligned}
V_{0}(\rho, \phi) & =\frac{\omega B_{1}}{2}\left(\rho^{2}-a^{2}-c \rho \cos \phi\right) \text { in Region II } \\
V_{0}(\rho, \phi) & =\frac{\omega B_{1} a^{2}}{2} \mathcal{V}(c, \rho, \phi) \text { in Region I , }
\end{aligned}
$$

where we defined,

$$
\mathcal{V}(u, \rho, \phi) \equiv \frac{u(\rho \cos \phi-u)}{\rho^{2}+u^{2}-2 u \rho \cos \phi} .
$$

Now we have the condition that the radial component of the current density vanishes on the disk-vacuum boundary: $J_{\rho}(\rho=A, \phi)=0$, or $\left.E_{\rho}\right|_{\rho=A}=0$, where $E(\vec{r})$ is the electric field inside the disk, from the known charges and from the charges at the boundary $\rho=A$. In terms of the potential $V(\vec{r})(\vec{E}=-\vec{\nabla} V)$ this condition reads $\partial V /\left.\partial \rho\right|_{\rho=A}=0$. This boundary condition can be fit if we consider an image cylindrical shell (with boundary shown as a dashed circle in Fig. 1) with charge density $-\left(A^{2} / c^{2}\right) \sigma_{N U}\left(\theta^{\prime}\right)$ and center at $O^{\prime}=\left(x=A^{2} / c, y=0\right)$, with $\theta^{\prime}$, and the corresponding radius $s^{\prime}$, as shown in Fig. 1. The potential inside the conducting disk due to this image charge is identical with that of the induced charge density $\sigma_{A}(\phi)$.

Thus, by adding the potential due to this image charge to $V_{0}(\rho, \phi)$ we obtain the potential $V(\rho, \phi)$ inside the disk:

$$
\begin{array}{cc}
V(\rho, \phi)= & \frac{\omega B_{1}}{2}\left[\left(\rho^{2}-a^{2}-c \rho \cos \phi\right)-\right. \\
& \left.-a^{2} \mathcal{V}\left(A^{2} / c, \rho, \phi\right)\right] \text { in Region II } \\
V(\rho, \phi)= & \frac{\omega B_{1} a^{2}}{2}\left[\mathcal{V}(c, \rho, \phi)-\mathcal{V}\left(A^{2} / c, \rho, \phi\right)\right] \\
\text { in Region I . }
\end{array}
$$

From the continuity of the potential at the boundary $\rho=A$, we obtain, in the region III outside the cylinder, the potential given by:

$$
\begin{gathered}
V(\rho, \phi)=\left(\frac{\omega B_{1} a^{2}}{2}\right)\left\{\frac{\rho^{2}-c^{2}}{\rho^{2}+c^{2}-2 c \rho \cos \phi}\right\} \\
\text { in Region III . }
\end{gathered}
$$

The discontinuity in the derivative of the potential at $\rho=A$ gives the charge density at the cylinder surface:

$$
\sigma_{A}(\phi)=\left(\epsilon_{0} \omega B_{1} a^{2} c\right) \frac{\left(A^{2}+c^{2}\right) \cos \phi-2 c A}{\left(A^{2}+c^{2}-2 c A \cos \phi\right)^{2}}
$$

The net surface charge is zero.

From $V$ we can calculate the current density in the disk through Ohm's law, $\vec{J}(\vec{r})=\sigma\left(-\vec{\nabla} V(\vec{r})+\omega B_{1}(\vec{r}) \rho \hat{\rho}\right)$. These expressions can be obtained straightforwardly but are too long and will not be shown here. Plots of the field lines of $\vec{J}(\rho, \phi)$ can be seen in Refs. $[11,17,19]$.

Now we consider the presence of another magnetic pole with uniform magnetic field $\vec{B}_{2}=B_{2} \hat{z}$ inside the circular region of radius $a$, with center at the distance $c_{2}$ located at $\left(x=c_{2} \cos \alpha, y=c_{2} \sin \alpha\right)$. The expressions for the electric potential due to this pole can be obtained from Eqs. 9 just replacing $B_{1}$ for $B_{2},, c$ for $c_{2}, \cos \phi$ for $\cos (\phi-\alpha)$ and $\sin \phi$ for $\sin (\phi-\alpha)$. Plots of the field lines of the total current $\vec{J}=\vec{J}_{1}+\overrightarrow{J_{2}}$ for an azimuthal arrangement of the poles with $c_{1}=c_{2}=c$ can be seen in Ref. [11] $(\alpha=\pi / 2)$ and in Ref. [19] ( $\alpha=\pi)$.

The flow of the eddy current due to one magnetic pole under its own magnetic field and also under the field of the other pole will generate braking torques on the disk, which are the focus of our analysis. These torques will be calculated on the next section.

\subsubsection{The torques}

The torque $\vec{T}_{n k}$ due to the flow of the current $\vec{J}_{n}$ (induced by the field $\vec{B}_{n}$ of pole $n$ ) under the field $\vec{B}_{k}$ of pole $k$ can be obtained through:

$$
\begin{array}{r}
\vec{T}_{n k}=\int \vec{r} \times\left(\vec{J}_{n} \times \vec{B}_{k}\right) d v= \\
-\hat{z} h B_{k} \int_{\text {Region II of pole } k} \rho^{2} J_{n \rho}(\rho, \phi) d \rho d \phi .
\end{array}
$$

We obtain

$$
T_{n n}=-\frac{\pi}{2} h \sigma B_{n}^{2} \omega a^{2} c^{2}\left[1-f\left(A^{2} / c\right)\right],
$$

where we defined the dimensionless function,

$$
f(u) \equiv \frac{a^{2} u}{c(u-c)^{2}} .
$$

Therefore, the braking torque $T_{n n}$ is given by:

$$
T_{n n}=-\frac{\pi}{2} h \sigma B_{n}^{2} \omega a^{2} c^{2}\left[1-\frac{A^{2} a^{2}}{\left(A^{2}-c^{2}\right)^{2}}\right],
$$

in agreement with the formula obtained by other authors $[17,19,20]$.

From the condition $d T_{n n} / d c=0$ we can show that, for the case $A>>a$, the maximum value of $T_{n n}$ occurs at $c=\tilde{c}$ with

$$
\tilde{c}=A\left[1-\left(\frac{a}{2 A}\right)^{2 / 3}\right] \quad(A>>a) .
$$


For $A / a=10$, for example, the exact location of the peak of $T_{n n}$ is at $c \simeq 0.861 A$, while the estimate above gives $\tilde{c} \simeq 0.864 A$. The maximum torque is given by:

$$
\begin{gathered}
T_{n n}^{(\max )}=T_{n n}(c=\tilde{c})= \\
-\frac{\pi}{2} h \sigma B_{n}^{2} \omega A^{2} a^{2}\left[1-3\left(\frac{a}{2 A}\right)^{2 / 3}\right] \quad(A>>a) .
\end{gathered}
$$

This behavior of $T_{n n}$ is in good agreement with experimental results (see, for example, Ref. [20]), as we show in Fig. 2. The values of the ratio $T_{n n} / T_{n n}^{(\max )}(\times 100)$ were extracted from an experimental curve [20] of the braking torque on a copper disk with $\sigma=57 \times 10^{6}(1 / \Omega \mathrm{m})$, $h=35 \times 10^{-6} \mathrm{~m}$, and $A=0.15 \mathrm{~m}$. Due to the spread of the pole magnetic field inside the air gap, the effective radius $a$ of the magnetic pole is determined through a best fit to the data. We obtained the value $a=0.030 \mathrm{~m}$ for this experimental setup (the crude theoretical estimate in terms of the pole magnetic flux is $a=0.018 \mathrm{~m} \mathrm{[20]).}$



Figure 2: Comparison of theoretical (Eq. 15) and measured results [20] for the ratio $T_{n n} / T_{n n}^{(\max )}$ versus the dimensionless radial distance $c / A$ of the pole center.

For the torque due to the interaction between two poles at arbitrary positions, one with center at a distance $c_{n}=c$ and the other with center at a distance $c_{k}$, along two radii separated by an angle $\alpha$, we obtain:

$$
\begin{array}{r}
T_{n k}\left(\alpha, c, c_{k}\right)=-\frac{\pi}{2} h \sigma B_{n} B_{k} \omega a^{2} c c_{k}[g(c, \alpha)- \\
\left.-g\left(A^{2} / c, \alpha\right)\right] \quad(n \neq k),
\end{array}
$$

in which

$$
g(u, \alpha) \equiv \frac{a^{2} u\left(\left(u^{2}+c_{k}^{2}\right) \cos \alpha-2 u c_{k}\right)}{c\left(u^{2}+c_{k}^{2}-2 u c_{k} \cos \alpha\right)^{2}} .
$$

For practical reasons, we consider separately two particular cases for the arrangement of the poles. The azimuthal arrangement $\left(c_{k}=c\right)$ and the radial arrangement $(\alpha=0)$.

For the torque due to the interaction between the poles at the azimuthal arrangement we obtain:

$$
\begin{aligned}
& T_{n k}(\alpha)=\frac{\pi}{4} h \sigma B_{n} B_{k} \omega a^{4}\left[\frac{1}{1-\cos \alpha}+\right. \\
& \left.+2 A^{2} c^{2} \frac{\left(A^{4}+c^{4}\right) \cos \alpha-2 A^{2} c^{2}}{\left(A^{4}+c^{4}-2 A^{2} c^{2} \cos \alpha\right)^{2}}\right]
\end{aligned}
$$

Note that in this equation, besides $n \neq k$, we have $\alpha_{\min } \leq \alpha \leq \pi$, with $\alpha_{\min }$ being the value of the displacement angle $\alpha$ for which the two poles touch each other:

$$
\cos \alpha_{\min }=1-2\left(\frac{a}{c}\right)^{2} .
$$

For the poles touching each other we can expect in practice a distortion in the pattern of eddy-current paths due to the attraction or repulsion of the two induced currents. This effect is more intense for the azimuthal arrangement of the poles and should change the braking torques in this case. In the low-speed regime, where the magnetic field due to the eddy currents is negligible, this repulsion or attraction is a very small effect and can also be neglected.

This expression of $T_{n k}(\alpha)$ is in agreement with the results we can find in literature for the cases $\alpha=\pi[11,19]$ and $\alpha=\pi / 2$ [11]. Eq. 20 can be found in Ref. [23], but there is no hint in this reference about how it was obtained.

For the radial arrangement of the poles we obtain:

$$
\begin{array}{r}
T_{n k}\left(c, c_{k}\right)=-\frac{\pi}{2} h \sigma B_{n} B_{k} \omega a^{2} c c_{k}\left[\frac{a^{2}}{\left(c-c_{k}\right)^{2}}-\right. \\
\left.-\frac{A^{2} a^{2}}{\left(A^{2}-c c_{k}\right)^{2}}\right],
\end{array}
$$

with $\left|c-c_{k}\right| \geq 2 a$ (poles without intersection).

The torque $T_{n k}(\alpha)$ adds to the torque $T_{n n}$ (both are along $-\hat{z}$ ) when the two poles have anti-parallel magnetic fields while for the torque $T_{n k}\left(c, c_{k}\right)$ this occurs when the poles are parallel. These mutual torques decay with the square of the distance between the poles, being maximum when the two poles are touching each other: $\alpha=\alpha_{\text {min }}$ for $T_{n k}(\alpha)$ and $\left|c-c_{k}\right|=2 a$ for $T_{n k}\left(c, c_{k}\right)$.

Fig. 3 shows the behavior of the braking factors $-T_{n n} / h \sigma B_{n}^{2} \omega,-T_{n k}\left(\alpha_{\min }\right) / h \sigma B_{n} B_{k} \omega$ for anti-parallel poles and $-T_{n k}(c, c-2 a) / h \sigma B_{n} B_{k} \omega$ for parallel poles as functions of the distance $c$ for fixed $A / a=10$. We can see that all the torques attain a maximum for certain values of the distance $c$ near the limit value $c=A-a$.

The maximums of the mutual torques are near the maximum of $T_{n n}$ given in Eq. 17. For the conditions shown in Fig. 3 we obtain that the maximum of $T_{n k}\left(\alpha_{\min }\right)$ occurs at $c \simeq 0.874 A$ while the maximum of $T_{n k}(c, c-2 a)$ occurs at $c \simeq 0.881 A$.

Fig. 4 shows the total torque per pole $T=\left(T_{11}+T_{22}+2 T_{12}\right) / 2$ for two poles touching each other as a function of the parameter $c / A$. We assume $B_{n}=B=-B_{k}$ for the plot of $T\left(\alpha_{\min }\right)=$ $\left[T_{11}(c)+T_{22}(c)+2 T_{12}\left(\alpha_{\min }\right)\right] / 2$, which has a peak at $\tilde{c}_{1} \simeq 0.864 A$ and $B_{n}=B=B_{k}$ for the plot of $T(c, c-$ 


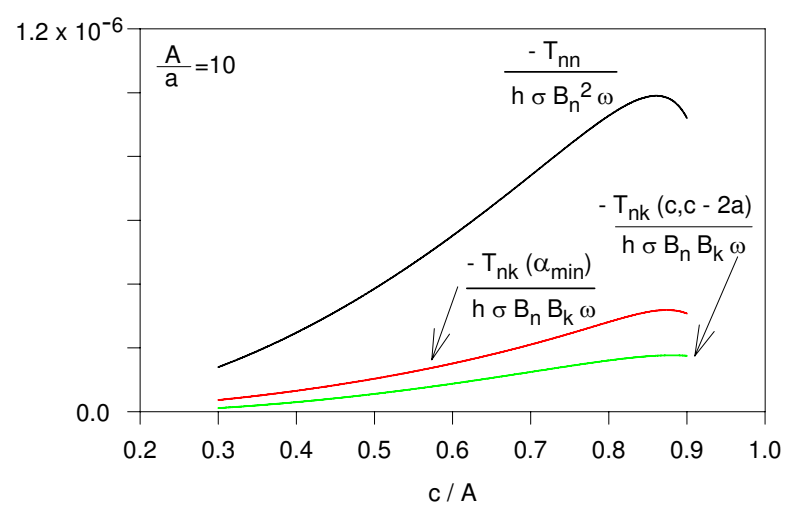

Figure 3: Braking factors $-T_{n n} / h \sigma B_{n}^{2} \omega$, $-T_{n k}\left(\alpha_{\min }\right) / h \sigma B_{n} B_{k} \omega$ and $-T_{n k}(c, c-2 a) / h \sigma B_{n} B_{k} \omega$ for two poles touching each other as functions of the ratio $c / A$ for $A / a=10$.

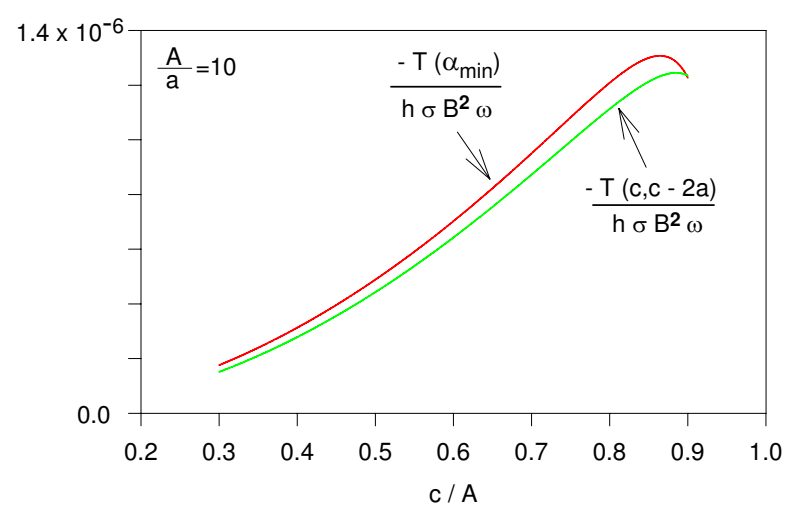

Figure 4: Ratios $-T\left(\alpha_{\min }\right) / h \sigma B^{2} \omega$ and $-T(c, c-$ $2 a) / h \sigma B^{2} \omega$ for the total torque per pole as functions of the ratio $c / A$ for the two poles touching each other at the azimuthal (red curve) and radial (green curve) arrangements.

$2 a)=\left[\left(T_{11}(c)+T_{22}(c-2 a)+2 T_{12}(c, c-2 a)\right] / 2\right.$ which has a peak at $\tilde{c}_{2} \simeq 0.885 \mathrm{~A}$. We can see that the azimuthal arrangement provides a larger torque than the radial arrangement of the two poles with exception of the region $c \simeq A-a$, where there is a small reversion of this behavior and the radial arrangement provides more torque than the azimuthal one.

\subsection{A rotating conducting ring}

Now we consider the effects on the braking torque due to a hollow cylinder. We consider a rotating conducting ring with external radius $A$ and internal radius $b$ (width $A-b$ ). For the pole with center at $(x=c, y=0)$, the condition that the radial component of the current density vanishes on the disk-vacuum boundaries $\left(J_{\rho}(\rho=A, \phi)=J_{\rho}(\rho=\right.$ $b, \phi)=0$ ) can be fit by means of an infinite series of image charges defined on cylinders centered at the positions $\left(x_{n}=A_{n}, y=0\right)$ and $\left(x_{n}=b_{n}, y=0\right)$ with surface charge densities $\sigma_{n}=(-1)^{n}\left(x_{n} / c\right) \sigma_{N U}\left(\theta_{n}^{\prime}\right)$. The positions $A_{n}$ (for the external images, $A_{n}>A$ ) and $b_{n}$ (for the internal images, $b_{n}<b$ ) are given by

$$
\begin{array}{r}
A_{n}=A^{2} / b_{n-1} \quad \text { and } \quad b_{n}=b^{2} / A_{n-1} \\
\text { for } n \geq 1,
\end{array}
$$

with $A_{0}=b_{0}=c$.

To calculate the torques we add the effects of this infinite series of image charges. We start defining the functional

$$
S[f(u)] \equiv \sum_{n=1}^{\infty}(-1)^{n}\left[f\left(A_{n}\right)+f\left(b_{n}\right)\right] .
$$

Therefore, the torque $T_{n n}$ for a rotating ring is given by:

$$
T_{n n}(b)=-\frac{\pi}{2} h \sigma B_{n}^{2} \omega a^{2} c^{2}\{1+S[f(u)]\},
$$

with $f(u)$ given in Eq. 14.

For the mutual torques we obtain:

$$
\begin{array}{r}
T_{n k}(b, \alpha)=-\frac{\pi}{2} h \sigma B_{n} B_{k} \omega a^{2} c^{2}\{g(c, \alpha)+ \\
+S[g(u, \alpha)]\}
\end{array}
$$

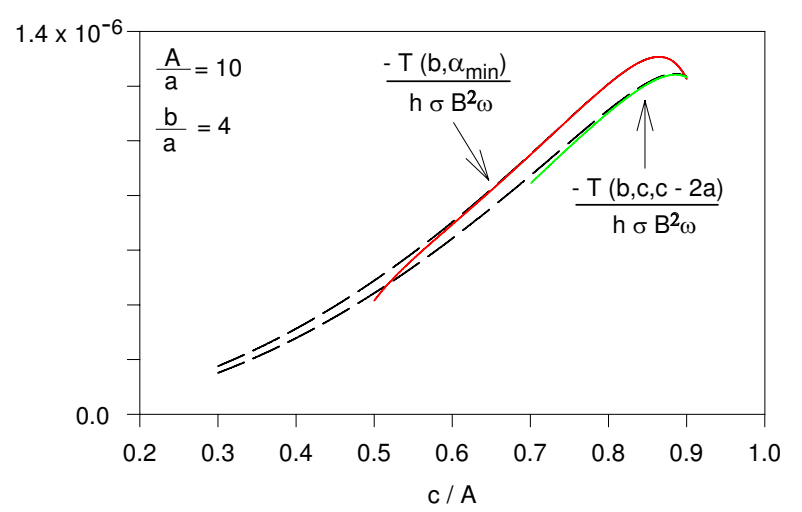

Figure 5: Ratios $\left.-T\left(b, \alpha_{\min }\right)\right) / h \sigma B_{n} B_{k} \omega$ and $-T(b, c, c-2 a)) / h \sigma B_{n} B_{k} \omega$ for the total torques per pole as functions of the ratio $c / A$ for $A / a=10$ and $b / a=4$. The two poles touch each other at the azimuthal (red curve) and radial (green curve) arrangements. The dashed black curves correspond to the case $b=0$, as shown in Fig. 4.

with $g(u, \alpha)$ given in Eq. 19, and

$$
\begin{array}{r}
T_{n k}\left(b, c, c_{k}\right)=-\frac{\pi}{2} h \sigma B_{n} B_{k} \omega a^{2} c c_{k}\{g(c, 0)+ \\
+S[g(u, 0)]\} .
\end{array}
$$

Fig. 5 shows the behavior of the total torque per pole for the azimuthal and radial arrangements with the two poles touching each other and for fixed $A / a=10$ and $b / a=4$. Comparing these curves with the ones for $b=0$ (dashed 


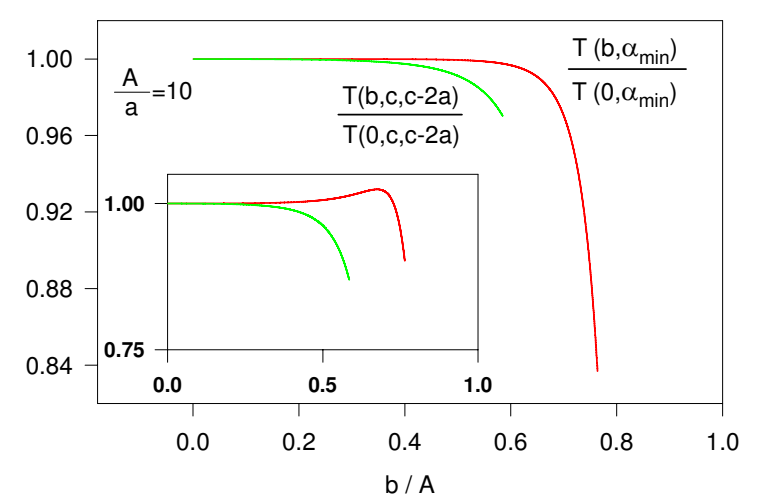

Figure 6: Ratios $T\left(b, \alpha_{\min }\right) / T\left(0, \alpha_{\min }\right)$ (red curve) for $c=\tilde{c}_{1} \simeq 0.864 A$ and $\alpha=\alpha_{\min } \simeq 0.232 \mathrm{rad}$ and $T(b, c c-2 a) / T(0, c, c-2 a)$ (green curve) for $c=\tilde{c}_{2} \simeq$ $0.885 A$ for the total torques per pole as functions of the ratio $b / A$ for $A / a=10$. The inset shows the ratios $T_{n k}\left(b, \alpha_{\min }\right) / T_{n k}\left(0, \alpha_{\min }\right)$ (red curve) and $T_{n k}(b, c, c-$ $2 a) / T_{n k}(0, c, c-2 a)$ (green curve) versus the ratio $b / A$.

black curves) we can see that at the region of maximum torques, $c \simeq \tilde{c}$, the torques are little affected by the presence of the inner radius $b$.

Figure 6 shows the ratios for the total torque per pole $T\left(b, \alpha_{\min }\right) / T\left(0, \alpha_{\min }\right)$ and $T(b, c, c-2 a) / T(0, c, c-2 a)$ versus the ratio $b / A$ for fixed $c$ (at the values $c=\tilde{c}_{1}$ and $c=\tilde{c}_{2}$ which correspond to the maximum torques) and $A / a=10$. We can see that as the inner radius $b$ approaches the limit $\tilde{c}_{1}-a$ for $T\left(b, \alpha_{\min }\right)$ and $\tilde{c}_{2}-3 a$ for $T(b, c, c-2 a)$ the torques fall very quickly. The narrower the ring, the more restricted are the current paths and the weaker the braking torque. At the inset we show the behavior of the similar ratios for the mutual torques $T_{n k}\left(b, \alpha_{\min }\right)$ and $T_{n k}(b, c, c-2 a)$. We can see that $T_{n k}\left(b, \alpha_{\min }\right)$ is not a monotonous function of $b / A$, it has a peak at $b \simeq 0.675 A$. A narrower ring restricts but also channel the current paths of one pole into the other and the mutual torque can be maximized. This behavior can not be used for optimization purposes because the total torque $T\left(b, \alpha_{\min }\right)$ is a monotonous function of $b / A$.

\subsection{A translating conducting strip}

Now we consider a very long conducting strip of width $\delta$ and thickness $h$ moving with constant velocity $v$ along $y$. The two poles with radius $a$ are located in space according to Fig. 7: $l$ and $t$ are the longitudinal and transverse displacements between the poles. Note that in this case the charge densities inside the sheet due to pole 1 are $\rho_{F}=\sigma_{U}=0$ and $\sigma_{N U}(\theta)=\epsilon_{0} v B_{1} \cos (\theta)$.

This case can be treated easily within the framework of the preceding calculations; we take the limit $b \rightarrow \infty$ with $A=b+\delta$. We also take $\cos \alpha=1-l^{2} /\left(2 c^{2}\right), c=b+x$ and $c_{k}=c+t$. Also, the constant velocity $v$ of the strip is $v=\omega c$ and the force is related to the torque we calculated

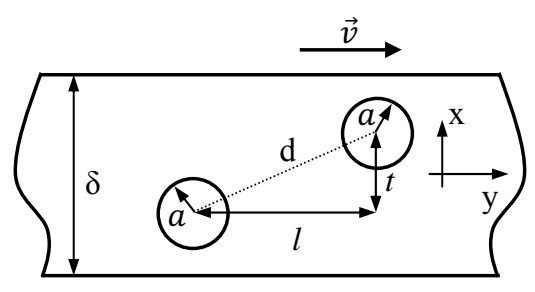

Figure 7: Plan view of the conducting strip of width $\delta$ moving along $y$ with the two poles with centers separated by a distance $d$.

before through $T=F c$. At this limit the image charges of the pole at $c$ are located at $A_{n}=c+n \delta$ and $b_{n}=c-n \delta$ and we obtain:

$$
S[f(u)]=2\left(\frac{a}{\delta}\right)^{2} \sum_{n=1}^{\infty} \frac{(-1)^{n}}{n^{2}},
$$

which is independent of the pole position $x$.

It follows that from the torque $T_{n n}$ we obtain the braking force on the strip along $y$ :

$$
F_{n n}(\delta)=-\frac{\pi}{2} h \sigma B_{n}^{2} v a^{2}\left[1-\frac{\pi^{2}}{6}\left(\frac{a}{\delta}\right)^{2}\right],
$$

which agrees with the result of Schieber [18] (deduced for $x=\delta / 2$ ).

For the force due to the interaction between the poles we obtain:

$$
\begin{aligned}
F_{n k}(l, t, \delta)=-\frac{\pi}{2} h \sigma B_{n} B_{k} v a^{2} & \left\{a^{2} \frac{t^{2}-l^{2}}{\left(t^{2}+l^{2}\right)^{2}}+\right. \\
& +S[g(u, \alpha)]\},
\end{aligned}
$$

where,

$$
\begin{array}{r}
S[g(u, \alpha)]=2 a^{2} \sum_{n=1}^{\infty}(-1)^{n}\left\{\frac{(n \delta-t)^{2}-l^{2}}{\left[(n \delta-t)^{2}+l^{2}\right]^{2}}+\right. \\
\left.+\frac{(n \delta+t)^{2}-l^{2}}{\left[(n \delta+t)^{2}+l^{2}\right]^{2}}\right\} .
\end{array}
$$

For practical reasons, we consider two simple pole arrangements: i) $t=0$ : the longitudinal arrangement, for which the two magnetic poles with radius $a$ have centers along the center line of the strip, with a displacement $l \geq 2 a$ between them; ii) $l=0$ : the transverse arrangement, where the two magnetic poles with radius $a$ have centers along the strip width, with a displacement $t \geq 2 a$ between them.

$$
\begin{gathered}
F_{n k}(l, \delta)=\frac{\pi}{2} h \sigma B_{n} B_{k} v a^{2}\left[\left(\frac{a}{l}\right)^{2}-\right. \\
\left.-2 a^{2} \sum_{n=1}^{\infty}(-1)^{n} \frac{n^{2} \delta^{2}-l^{2}}{\left(n^{2} \delta^{2}+l^{2}\right)^{2}}\right] .
\end{gathered}
$$


Analogously, for the linear transverse arrangement:

$$
\begin{gathered}
F_{n k}(t, \delta)=-\frac{\pi}{2} h \sigma B_{n} B_{k} v a^{2}\left[\left(\frac{a}{t}\right)^{2}+\right. \\
\left.+2 a^{2} \sum_{n=1}^{\infty}(-1)^{n} \frac{n^{2} \delta^{2}+t^{2}}{\left(n^{2} \delta^{2}-t^{2}\right)^{2}}\right] .
\end{gathered}
$$

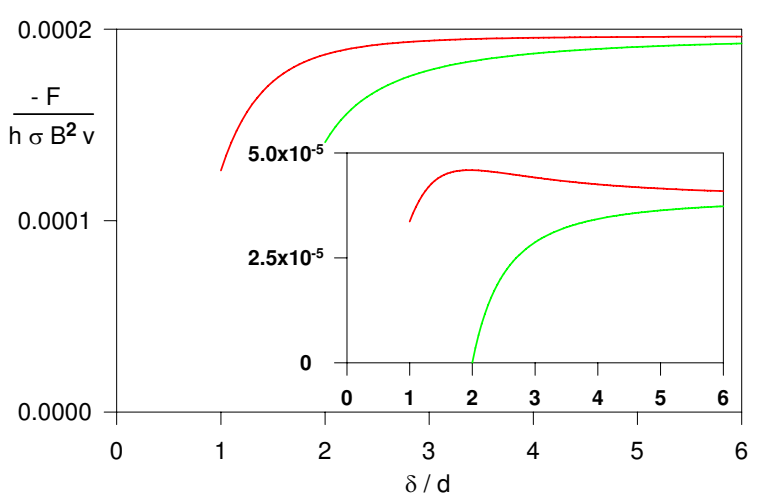

Figure 8: Braking factor $-F / h \sigma B^{2} v$ as a function of the ratio $\delta / d$ for poles touching each other $(d / a=2)$ : longitudinal (red curve) and transverse (green curve) arrangements. At the inset we show the behavior of the similar ratios for the mutual forces $F_{n k}(l=d, \delta)$ (red curve) and $F_{n k}(t=d, \delta)$ (green curve) versus $\delta / d$.

The behavior of the total braking force per pole $F=$ $F_{n n}+F_{n k}$ for the two arrangements versus the ratio $\delta / d$ for a fixed distance $d=2 a$ between the poles (poles touching each other) is shown in Fig. 8. We can see that the force is larger for the longitudinal arrangement. The inset shows the behavior of the mutual forces $F_{n k}(l=d, \delta)$ and $F_{n k}(t=d, \delta)$ versus $\delta / d$ for the two poles touching each other. We can see that, analogously to the mutual torque $T_{n k}\left(b, \alpha_{\min }\right)$, the force $F_{n k}(l=d, \delta)$ is not monotonous and has a peak at $\delta / d \simeq 1.95$. In contrast, the total force is always a monotonous function of $\delta / d$. The peak in $F_{n k}(l=d, \delta)$ can lead to the design of a break with maximum force only in the case of an unreal infinite linear array of poles [24].

To illustrate the above results, we take a numerical example with practical magnitudes (based in Ref. [20]): $\sigma=3.4 \times 10^{7} 1 / \Omega \mathrm{m}$ (copper), $h=2 \mathrm{~mm}, a=1 \mathrm{~cm}$, $v=0.05 \mathrm{~m} / \mathrm{s}, B_{n}=B_{k}=0.75 \mathrm{~T}$ and $\delta=5 \mathrm{~cm}$, for which we obtain $F_{n n} \simeq 0.281 \mathrm{~N}$. In this case, for two touching poles $(d=2 \mathrm{~cm})$ we obtain $F_{n k}(l=d, \delta) \simeq 0.0864 \mathrm{~N}$ and $F_{n k}(t=d, \delta) \simeq 0.0405 \mathrm{~N}$.

\section{Conclusions}

We have presented a theory of magnetic braking based on the analytical calculation of the charge densities induced on a moving conductor. Through electrostatics and magnetostatics we derived analytical expressions for the braking torques and forces in an unified formalism, valid for rotating and linear magnetic brakes with round poles and low operating speeds, when the magnetic field due to the induced currents is negligible in comparison with the external field.

Our analysis recover some analytical results for the forces and torques derived in some previous works on the magnetic brake and extend these results for the rotating ring geometry and for the case of an arbitrary arrangement of magnetic poles for linear and rotating brakes. In general, the rotating azimuthal and the linear longitudinal arrangements have better braking performances than the radial and transverse ones respectively.

These analytical results are important to provide insight into the fundamental physics of eddy-current braking and to establish preliminary design data, such as the size and pole positions for maximum braking torque and force which are compatible with the envisioned application of the braking system. Unfortunately, these results are not always available and they can be obtained only for simple geometries and specific conditions, such as the low angular speed and stationary regimes. For more complex systems, numerical methods such as the finite element (FEM), the boundary element (BEM) or the coupled FEM-BEM methods can be implemented into a computer program (see, for example, $[25,26])$. The analytical expressions for the electric potential or current density we obtain here can provide a sufficiently accurate initial guess for the application of these iterative numerical processes in more complex systems.

In practice, the use of multiple magnetic poles can be justified in order to increase the total torque or to avoid undesired unbalanced forces and torques on the moving conductor. The ring geometry, in turn, is a prevailing geometry on rotating brakes due to the central axle hole and it has the advantage over the disk geometry in the easily adjustable moment of inertia for a fixed distance $c$ of the pole center.

Since the studied system involves moving components (a disk, a ring or a plate), a complete time-domain modeling would require the coupling between the current/torque equations and the mechanical ones, e.g., Newton's second law for rotation/translation. The mechanical parameters involved in these equations, such as mass and moment of inertia, can have a crucial influence in the system's braking performance. We plan to investigate these extensions of the present analysis by examining the system's electromechanical transients in a future work.

\section{References}

[1] B. A. Knyazev, I. A. Kotel'nikov, A. A. Tyutin and V. S. Cherkassky, Braking of a magnetic dipole moving with an arbitrary velocity through a conducting pipe, Physics.-Usp 49 937-946, 2006.

[2] S. Land, P. McGuire, N. Bumb, B. P. Mann and B. B. Yellen, Electromagnetic braking revisited with a magnetic point dipole model, Am. J. Phys. 84, 257262, 2016. 
[3] X. Xie, Zhu-ying Wang, P. Gu, Zhi-jian Jian, Xiao-lin Chen and Z. Xie, Investigation of magnetic damping on an air track, Am. J. Phys. 74, 974978, 2006.

[4] A. Vidaurre, J. Riera, J. A. Monsoriu and M. H. Gim'enez, Testing theoretical models of magnetic damping using an air track, Eur. J. Phys. 29, 335343, 2008 .

[5] H. D. Wiederick, N. Gauthier, D. A. Campbell, and P. Rochon, Magnetic braking: Simple theory and experiment, Am. J. Phys. 55, 500503, 1987.

[6] P. Lorrain, Electrostatic charges in $\mathrm{v} \times \mathrm{B}$ fields: the Faraday disk and the rotating sphere, Eur. J. Phys. 11, 94-98, 1990.

[7] P. Lorrain, Electrostatic charges in $\mathrm{v} \times \mathrm{B}$ fields, Eur. J. Phys. 22, L3-L4, 2001 and D. V. Redžić, Comment on electrostatic charges in $\mathrm{v} \times \mathrm{B}$ fields, Eur. J. Phys. 22, L1-L2, 2001.

[8] P. Lorrain, J. McTavish and F. Lorrain, Magnetic fields in moving conductors: four simple examples, Eur. J. Phys. 19, 451-457, 1998.

[9] G. S. Smith, Faraday's first dynamo: A retrospective, Am. J. Phys. 81, 907-917, 2013.

[10] J. A. Redinz, Faraday's first dynamo: An alternate analysis, Am. J. Phys. 83, 179-182, 2015.

[11] J. A. Redinz, The induction motor, Eur. J. Phys. 36, $055008,2015$.

[12] P. J. Salzman, J. R. Burke, and S. M. Lea, The effect of electric fields in a classic introductory treatment of eddy current forces, Am. J. Phys. 69, 586590, 2001.

[13] M. A. Heald, Magnetic braking: Improved theory, Am. J. Phys. 56, 521522, 1988.

[14] M. Marcuso, R. Gass, D. Jones, and C. Rowlett, Magnetic drag in the quasi-static limit: A computational method, Am. J. Phys. 59, 11181123, 1991.

[15] K. Lee and K. Park, Modeling eddy currents with boundary conditions by using Coulomb's law and the method of images, IEEE Trans. on Mag. 38, 13331340, 2002.

[16] K. Lee and K. Park, Analysis on an eddy current brake considering finite radius and induced magnetic flux, $\mathrm{J}$. App. Phys. 92, 5532-5538, 2002.

[17] J. M. Aguirregabiria, A. Hernández and M. Rivas, Magnetic braking revisited, Am. J. Phys. 65, 851-856, 1997.

[18] D. Schieber, Unipolar induction braking of thin metal sheets, Proc. IEE 119, 1499-1503, 1972.

[19] W. R. Smythe, On eddy currents in a rotating disk, Trans. AIEE 61, 681-684, 1942.
[20] D. Schieber, Braking torque on rotating sheet in stationary magnetic field, Proc. IEE 121, 117-122, 1974.

[21] A. H. C. Gosline and V. Hayward, Eddy Current Brakes for Haptic Interfaces: Design, Identification, and Control, IEEE/ASME Trans. on Mechatronics 13, 669-677, 2008

[22] W. G. V. Rosser, An Introduction to the Theory of Relativity (Butterworth \& Co., London, 1964), 1 st ed.

[23] K. Venkataratnam and D. Ramachandra Raju, Analysis of eddy-current brakes with nonmagnetic rotors, Proc. IEE 124, 6771, 1977.

[24] D. Schieber, Force on a moving conductor due to a magnetic pole array, Proc. IEE 120, 1519-1520, 1973.

[25] Y. E. Lezhi, G. Yang and D. Li, Analytical model and finite element computation of braking torque in electromagnetic retarder, Front. Mech. Eng. 9, 368379, 2014.

[26] J. Camaño and R. Rodríguez, Analysis of a FEMBEM model posed on the conducting domain for the timedependent eddy current problem, J. Comput. Appl. Math. 236 3084-3100, 2012. 